\title{
DEFINITIONS FOR UNIFORM DOMAINS
}

\section{O. MARTIO}

\section{Introduction}

The concept of an $(\alpha, \beta)$-uniform domain $D$ in $R^{n}$ was introduced in [MS]. Although useful in several applications, this definition has no immediate conformally invariant meaning in $\bar{R}^{n}=R^{n} \cup\{\infty\}$. Here we present a very simple condition, called $\delta$-uniformity and based on the concept of the cross ratio, which turns out to be equivalent to the $(\alpha, \beta)$-uniformity in the case $D \subset R^{n}$.

A domain $D \subset \bar{R}^{n}$ is called $\delta$-uniform, $0<\delta \leqq 1$, if for all $x_{1}, x_{2} \in D, x_{1} \neq x_{2}$, there is a continuum $K$ in $D$ connecting $x_{1}$ to $x_{2}$ with

$$
\frac{|x-y|}{\left|x-x_{i}\right|} \frac{\left|x_{1}-x_{2}\right|}{\left|y-x_{j}\right|} \geqq \delta, \quad i \neq j, \quad i, j=1,2
$$

for all $x \in K \backslash\left\{x_{1}, x_{2}\right\}$ and $y \in \bar{R}^{n} \backslash D$.

In order to prove that a domain $D \subset R^{n}$ is $\delta$-uniform if and only if it is $(\alpha, \beta)$ uniform we consider two other definitions for the $(\alpha, \beta)$-uniformity in Chapters 3 and 4. The main equivalence is then proved in Chapter 5. Chapter 6 is devoted to some general properties of uniform domains, e.g. if $D$ is a $\delta$-uniform domain, the Hausdorff-dimension of $\partial D$ satisfies $\operatorname{dim}_{H} \partial D \leqq c<n$, where $c$ depends only on $\delta$ and $n$.

In $\bar{R}^{2}$ a simply connected domain $D$ is $\delta$-uniform if and only if $D=\bar{R}^{2}$ or $D=\bar{R}^{2} \backslash\{z\}$ or $D$ is a quasiconformal disc. In the case of Jordan domains this gives a new and simple characterization of quasiconformal discs in $\bar{R}^{2}$.

Notation will be as in [MS] and generally standard. A rectifiable path is always parametrized by means of arc length. If $\gamma:[a, b] \rightarrow \bar{R}^{n}$ is a path, then $|\gamma|=\gamma[a, b]$ is the locus of $\gamma$. 


\section{John domains and $(\alpha, \beta)$-uniform domains}

First we recall the definitions presented in [MS].

2.1. Definition. A domain $G \subset R^{n}$ is called an $(\alpha, \beta)$-John domain, $0<\alpha \leqq$ $\beta<\infty$, if there is $x_{0} \in D$ such that every $x \in D$ can be joined to $x_{0}$ by a rectifiable path $\gamma:[0, d] \rightarrow D$ such that $d \leqq \beta$ and

$$
\operatorname{dist}(\gamma(t), \partial D) \geqq \frac{\alpha}{d} t \quad \text { for } \quad t \in[0, d] .
$$

The point $x_{0}$ is called a center of $D$.

2.3. An alternative characterization for John domains was given in [MS, Lemma 2.7]. Here we shall need the following fact included in the proof of that lemma: Suppose that $G \subset R^{n}$ is a domain, $x, x_{0} \in G$ and $x$ can be joined to $x_{0}$ using a path $\gamma:[0,1] \rightarrow D$ such that for some $\delta \in(0,1]$

$$
\gamma[0, t] \subset B^{n}\left(\gamma(t), \frac{1}{\delta} \operatorname{dist}(\gamma(t), \partial G)\right), \quad 0 \leqq t \leqq 1 .
$$

Then there exists a rectifiable path $\gamma_{1}:[0, d] \rightarrow G$ joining $x$ to $x_{0}$ and satisfying

$$
\begin{gathered}
d \leqq\left|x-x_{0}\right| / \varphi^{2} \\
\operatorname{dist}\left(\gamma_{1}(t), \partial D\right) \geqq \varphi t, \quad t \in[0, d],
\end{gathered}
$$

and $\varphi$ depends only on $n$ and $\delta$.

2.7. Definition. A domain $D \subset R^{n}$ is called $(\alpha, \beta)$-uniform, $0<\alpha \leqq \beta<\infty$, if for each pair of points $x_{1}, x_{2} \in D, x_{1} \neq x_{2}$, there is an $\left(\alpha\left|x_{1}-x_{2}\right|, \beta\left|x_{1}-x_{2}\right|\right)$-John domain $G$ such that $x_{1}, x_{2} \in G \subset D$.

2.8. Remark. The definition for uniformity in the above sense can easily be extended for domains in $\bar{R}^{n}$; for instance, a domain $D \subset \bar{R}^{n}$ can be called $(\alpha, \beta)$ uniform if $D \cap R^{n}$ is $(\alpha, \beta)$-uniform in the sence of 2.7. However, we only consider $(\alpha, \beta)$-uniform domains in $R^{n}$ since the definition for $\delta$-uniform domains applies to $\bar{R}^{n}$ and reduces to $(\alpha, \beta)$-uniformity in $R^{n}$; see Chapter 5 .

\section{Domains of type $(\alpha, \beta)$}

3.1. Definition. A domain $D \subset R^{n}$ is of type $(\alpha, \beta), 0<\alpha \leqq \beta<\infty$, if for each pair of points $x_{1}, x_{2} \in D, x_{1} \neq x_{2}$, there is a rectifiable path $\gamma:[0, d] \rightarrow D$ joining $x_{1}$ to $x_{2}$ and

$$
d \leqq \beta\left|x_{1}-x_{2}\right|
$$

(3.3) $\operatorname{dist}(\gamma(t), \partial D) \geqq \frac{\alpha}{\beta} p_{d}(t), t \in[0, d], \quad$ where $\quad p_{d}(t)=d / 2-|t-d / 2|, t \in[0, d]$. 
3.4. Theorem. (a) If $D$ is $(\alpha, \beta)$-uniform, then $D$ is of type $(\alpha, 2 \beta)$. (b) If $D$ is of type $(\alpha, \beta)$, then $D$ is $\left((\alpha / \beta)^{2} / 32, \beta\right)$-uniform.

Proof. For (a) suppose that $D$ is $(\alpha, \beta)$-uniform. Let $x_{1}, x_{2} \in D, x_{1} \neq x_{2}$. Then there exist rectifiable paths $\gamma_{i}:\left[0, d_{i}\right] \rightarrow D$ joining $x_{i}$ to a point $x_{0}$ of $D$ and

$$
d_{i} \leqq \beta\left|x_{1}-x_{2}\right|, \quad \operatorname{dist}\left(\gamma_{i}(t), \partial D\right) \geqq \alpha\left|x_{1}-x_{2}\right| t / d_{i} .
$$

Since $\alpha\left|x_{1}-x_{2}\right| t / d_{i} \geqq \alpha t / \beta$, the composed path $\gamma=\gamma_{2}^{-1} \gamma:\left[0, d_{1}+d_{2}\right] \rightarrow D$ clearly satisfies (3.2) and (3.3) for the required $\alpha$ and $\beta$.

For (b) assume that $D$ is of type $(\alpha, \beta)$. Let $x_{1}, x_{2} \in D, x_{1} \neq x_{2}$, and let $\gamma$ be a path as in 3.1. Write for $t>0$

$$
A_{t}=\bigcup_{s \in(0, d)} B^{n}\left(\gamma(s), t p_{d}(s)\right)
$$

Then $A_{t}$ is a domain in $D$ provided $t \in(0, \alpha / \beta)$.

Let $\varepsilon=(1 / 4) \min \left(d\right.$, dist $\left.\left(A_{\alpha / 2 \beta}, \partial D\right)\right)>0$. Set $G=A_{\alpha / 2 \beta}+B^{n}(\varepsilon)$. We claim that $G$ is an $\left(\alpha^{\prime}, \beta^{\prime}\right)$-John domain with

$$
\alpha^{\prime}=(\alpha / \beta)^{2}\left|x_{1}-x_{2}\right| / 32, \quad \beta^{\prime}=\beta\left|x_{1}-x_{2}\right|
$$

and $x_{1}, x_{2} \in G \subset D$. The last two assertions are trivial and it remains to prove the first.

Set $x_{0}=\gamma(d / 2)$ and let $y \in G$. Fix $s \in[0, d]$ such that $y \in B^{n}\left(\gamma(s), \alpha p_{d}(s) / 2 \beta\right)+$ $B^{n}(\varepsilon)$. By symmetry we may assume $s \leqq d / 2$. Let $\gamma_{1}:\left[0, d_{1}\right] \rightarrow G$ represent a straight line segment joining $y$ to $\gamma(s)$. The composed path $\gamma_{2}=(\gamma \mid[s, d / 2]) \gamma_{1}$ joins $y$ to $x_{0}$ in $G$. Let $\gamma_{2}:[0, l] \rightarrow G$ with arc length as parameter. Now

$$
l \leqq d_{1}+d / 2 \leqq \alpha s / 2 \beta+\varepsilon+d / 2 \leqq \alpha d / 4 \beta+\varepsilon+d / 2 \leqq d \leqq \beta\left|x_{1}-x_{2}\right| .
$$

Choose $t \in[0, l]$. If $t \in\left[0, d_{1}\right]$, then clearly

$$
\operatorname{dist}\left(\gamma_{2}(t), \partial G\right) \geqq t / 2 \geqq \alpha t / 2 \beta \text {. }
$$

Suppose $t>d_{1}$. Pick $s^{\prime} \in[s, d / 2]$ with $d / 2-s^{\prime}=l-t$. Since $l-d / 2 \leqq \varepsilon$, the inequality $s^{\prime}+\varepsilon \geqq t$ holds. If $t / 2 \geqq \varepsilon$,

$$
\operatorname{dist}\left(\gamma_{2}(t), \partial G\right)=\operatorname{dist}\left(\gamma\left(s^{\prime}\right), \partial G\right) \geqq \frac{\alpha}{2 \beta} s^{\prime} \geqq \frac{\alpha}{2 \beta}(t-\varepsilon) \geqq \frac{\alpha}{4 \beta} t .
$$

On the other hand, if $t / 2<\varepsilon$, then

$$
\operatorname{dist}\left(\gamma_{2}(t), \partial G\right) \geqq \varepsilon+\frac{\alpha}{2 \beta} s^{\prime} \geqq \frac{\alpha}{2 \beta}\left(\varepsilon+s^{\prime}\right) \geqq \frac{\alpha}{2 \beta} t .
$$

The conclusion now follows from

3.8. Lemma. Suppose that $G \subset R^{n}$ is a domain and $x_{0} \in G$. If there exist numbers $0<\alpha \leqq \beta<\infty$ such that every point $x \in G$ can be joined to $x_{0}$ using a rectifiable path $\gamma:[0, d] \rightarrow G$ with $d \leqq \beta$ and

$$
\operatorname{dist}(\gamma(t), \partial G) \geqq \frac{\alpha}{\beta} t
$$

then $G$ is an $\left((\alpha / \beta)^{2} \operatorname{dia}(G) / 2, \beta\right)$-John domain. 
3.10. Remark. The only difference is in the lower bounds of (2.2) and (3.9).

Proof for Lemma 3.8. Set $p=\alpha \operatorname{dia}(G) / 2 \beta$. Clearly $\operatorname{dia}(G) \leqq 2 \beta$ and $B^{n}\left(x_{0}, p\right) \subset G$. Note also that $(\alpha / \beta)^{2}$ dia $(G) / 2 \leqq \beta$.

Let $x \in G$ and let $\gamma:[0, d] \rightarrow G$ be a path as in (3.9). If $d<p$, then instead of $\gamma$ we can use a straight line segment $\gamma^{\prime}$ connecting $x$ to $x_{0}$, and it is not difficult to see that

$$
\operatorname{dist}\left(\gamma^{\prime}(t), \partial G\right) \geqq p t / d^{\prime}, \quad t \in\left[0, d^{\prime}\right],
$$

where $d^{\prime}$ is the length of $\gamma^{\prime}$. On the other hand, if $d \geqq p$, then

$$
\operatorname{dist}(\gamma(t), \partial G) \geqq \alpha t / \beta=\alpha d t / \beta d \geqq(\alpha / \beta)^{2} \operatorname{dia}(G) t / 2 d .
$$

The lemma follows.

To finish the proof of (b) note that by (3.6) and (3.7)

$$
\operatorname{dist}\left(\gamma_{2}(t), \partial G\right) \geqq \frac{\alpha}{4 \beta} t=\frac{\alpha}{4 \beta} \frac{\left|x_{1}-x_{2}\right|}{\left|x_{1}-x_{2}\right|} t
$$

Since dia $(G) \geqq\left|x_{1}-x_{2}\right|$, Lemma 3.8 implies by (3.5) and (3.11) that $G$ is a $\left(32^{-1}(\alpha / \beta)^{2}\left|x_{1}-x_{2}\right|, \beta\left|x_{1}-x_{2}\right|\right)-J o h n$ domain. This completes the proof for (b).

\section{Condition $\mathbf{A}(\delta)$}

For uniform domains this condition is a counterpart of [MS, Lemma 2.7] given for John-domains.

4.1. Definition. A domain $D \subset R^{n}$ satisfies the condition $\mathrm{A}(\delta), \quad 0<\delta \leqq 1$, if for all $x_{1}, x_{2} \in D$ there is a path $\gamma:[0, s] \rightarrow D$ (not necessarily rectifiable) joining $x_{1}$ to $x_{2}$ and

$$
\operatorname{dia}|\gamma| \leqq\left|x_{1}-x_{2}\right| / \delta
$$

$$
\begin{array}{ll}
\gamma[0, t] \subset \bar{B}^{n}\left(\gamma(t), \frac{1}{\delta} \operatorname{dist}(\gamma(t), \partial D)\right), & 0 \leqq t \leqq s / 2 \\
\gamma[t, s] \subset \bar{B}^{n}\left(\gamma(t), \frac{1}{\delta} \operatorname{dist}(\gamma(t), \partial D)\right), & s / 2 \leqq t \leqq s .
\end{array}
$$

4.5. Theorem. (a) If a domain $D \subset R^{n}$ satisfies the condition $\mathrm{A}(\delta), 0<\delta \leqq 1$, then $D$ is of type $(\alpha, \beta)$, where $0<\alpha \leqq \beta<\infty$ depend only on $\delta$ and, possibly, on $n$. (b) If $D$ is of type $(\alpha, \beta)$, then $D$ satisfies $\mathrm{A}(\delta)$ with $\delta=\min (1 / \beta, \alpha / \beta)$.

Proof. The proof for (a) rests on 2.3. Suppose that $D$ satisfies the condition $\mathrm{A}(\delta)$. Let $x_{1}, x_{2} \in D$ and let $\gamma:[0, s] \rightarrow D$ be a path as in 4.1. We may assume 
$s=2$. Write $x_{0}=\gamma(1)$. By 2.3 there are rectifiable paths $\gamma_{i}:\left[0, d_{i}\right] \rightarrow D$ connecting $x_{i}$ to $x_{0}, i=1,2$, with

$$
\begin{gathered}
\operatorname{dist}\left(\gamma_{i}(t), \partial D\right) \geqq \varphi t \\
d_{i} \leqq\left|x_{i}-x_{0}\right| / \varphi^{2},
\end{gathered}
$$

where $\varphi \in(0,1]$ depends only on $\delta$ and $n$. Letting $\gamma=\gamma_{2}^{-1} \gamma_{1}$ it is not difficult to see that $\gamma$ satisfies (3.2) and (3.3) with $\beta=2 / \varphi^{2} \delta, \alpha=1$.

The proof for (b) is easy. Suppose that $D$ is of type $(\alpha, \beta)$. Pick $x_{1}, x_{2} \in D$, $x_{1} \neq x_{2}$, and let $\gamma:[0, d] \rightarrow D$ be a rectifiable path joining $x_{1}$ to $x_{2}$ as in (3.2) and (3.3). Now dia $(|\gamma|) \leqq d \leqq \beta\left|x_{1}-x_{2}\right|$ and for $t \in[0, d / 2]$

$$
\gamma[0, t] \subset \bar{B}^{n}(\gamma(t), t) \subset \bar{B}^{n}(\gamma(t),(\beta / \alpha) \operatorname{dist}(\gamma(t), \partial D)),
$$

and, by symmetry, the same holds for $\gamma[t, d]$ in the interval $[d / 2, d]$. Thus $\gamma$ satisfies (4.2)-(4.4) with $\delta=\min (1 / \beta, \alpha / \beta)$. The proof is complete.

\section{5. $\delta$-uniform domains}

5.1. Definition. A domain $D \subset \bar{R}^{n}$ is called $\delta$-uniform, $0<\delta \leqq 1$, if for all $x_{1}, x_{2} \in D, x_{1} \neq x_{2}$, there is a continuum $K$ joining $x_{1}$ to $x_{2}$ such that the cross ratio

$$
\left(x, y, x_{i}, x_{j}\right)=\frac{|x-y|}{\left|x-x_{i}\right|} \frac{\left|x_{i}-x_{j}\right|}{\left|x_{j}-y\right|} \geqq \delta, \quad i, j=1,2, i \neq j,
$$

for all $x \in K \backslash\left\{x_{1}, x_{2}\right\}$ and $y \in \complement D$.

5.3. Remark. The cross ratio $\left(x, y, x_{1}, x_{2}\right)$ is defined whenever all four points are distinct in $\bar{R}^{n}$. Especially, if $y=\infty$, then

$$
\left(x, \infty, x_{1}, x_{2}\right)=\left|x_{1}-x_{2}\right| /\left|x-x_{1}\right| .
$$

Observe that the cross ratio is a conformal invariant in $\bar{R}^{n}$. If $x, y, x_{1}, x_{2}$ are four distinct points of $\bar{R}^{n}$, then there is a Möbius transformation $T$ with $T\left(x_{1}\right)=0, T(y)=\infty$ and $T(x)=e_{1}$ and we have

$$
\left(x, y, x_{1}, x_{2}\right)=\left|T\left(x_{2}\right)\right| .
$$

5.4. Theorem. (a) If a domain $D \subset R^{n}$ is $\delta$-uniform, then $D$ is $(\alpha, \beta)$-uniform and $0<\alpha \leqq \beta<\infty$ depend only on $\delta$ and $n$.

(b) If $D \subset R^{n}$ is $(\alpha, \beta)$-uniform, then $D$ is $\delta$-uniform and $\delta \in(0,1]$ depends only on $\alpha$ and $\beta$.

Proof. For (a) fix $x_{1}, x_{2} \in D, x_{1} \neq x_{2}$, and let $K$ be a connecting continuum as in 5.1. Let $K_{1}$ be a subcontinuum of $K$ such that $K_{1}$ joins $S^{n-1}\left(x_{1}, s\right)$ to $S^{n-1}\left(x_{2}, s\right)$, $s=\left|x_{1}-x_{2}\right| / 4$, in $D \backslash \bigcup_{i=1,2} B^{n}\left(x_{i}, s\right)$. Choose $x_{0} \in K_{1} \cap S^{n-1}\left(x_{1}, s\right)$. 
By symmetry and trivial geometric considerations it suffices to show that $x_{1}$ can be joined to $x_{0}$ by a path $\varepsilon$ such that

$$
\varepsilon[0, t] \subset \bar{B}^{n}\left(\varepsilon(t), \frac{1}{\varkappa} \operatorname{dist}(\varepsilon(t), \partial D)\right)
$$

where $\varkappa$ depends on $\delta$ and $n$, since then it is easy to see that $D$ satisfies the $\mathrm{A}\left(\delta^{\prime}\right)$ condition and hence Theorems 4.5 and 3.4 show that $D$ is $(\alpha, \beta)$-uniform.

To construct the path $\varepsilon$ define a sequence of points $z_{0}, z_{1}, \ldots$ and paths $\gamma_{i}$ connecting $z_{i}$ to $z_{i+1}$ as follows. Set $z_{0}=x_{0}$. Choose $z_{1} \in K \cap S^{n-1}\left(x_{1}, s / 2\right)$. Since $D$ is $\delta$-uniform, there is a continuum connecting $z_{0}$ to $z_{1}$ in $D$ and satisfying (5.2). Without loss of generality we may assume that the continuum is a path. Call it $\gamma_{0}$. In general we pick $z_{i} \in S^{n-1}\left(x_{1}, s / 2^{i}\right) \cap K$ and define $\gamma_{i}$ similarly.

Fix $i$. For $y \in \complement D$ with $\left|z_{i}-y\right|=\operatorname{dist}\left(z_{i},\lceil D)\right.$ the estimate

$$
\operatorname{dist}\left(z_{i},[D) \geqq \delta s\left|x_{2}-y\right| /\left|x_{1}-x_{2}\right| 2^{i} \geqq \delta\left|x_{2}-y\right| / 2^{i+2}\right.
$$

holds. If now $\left|x_{2}-y\right|<\left|x_{1}-x_{2}\right| / 2$ for some $i$, then $\varepsilon$ can be chosen to be a straight line segment and the estimate (5.5) is trivial. Otherwise $\left|x_{2}-y\right| \geqq\left|x_{1}-x_{2}\right| / 2$ for all $i$. Consequently (5.6) yields

$$
\operatorname{dist}\left(z_{i},[D) \geqq \delta\left|x_{1}-x_{2}\right| / 2^{i+3}=r_{i}, \quad i=0,1, \ldots .\right.
$$

Consider $\gamma_{i}$. Let $x \in\left|\gamma_{i}\right| \backslash \bigcup_{j=i, i+1} B^{n}\left(z_{j}, r_{j} / 2\right)$. For $y \in \complement D$

$$
|x-y| \geqq \delta\left|x-z_{i}\right|\left|y-z_{i+1}\right| /\left|z_{i}-z_{i+1}\right| \text {. }
$$

On the other hand, $\left|x-z_{i}\right| \geqq r_{i} / 2,\left|y-z_{i+1}\right| \geqq r_{i+1}$ and $\left|z_{i}-z_{i+1}\right| \leqq 2 s / 2^{i}$; thus (5.8) yields

$$
|x-y| \geqq \delta^{3}\left|x_{1}-x_{2}\right| / 2^{i+7} \text {. }
$$

Clearly the same estimate holds if $x \in|\gamma| \cup \bigcup_{j=i, i+1} \bar{B}^{n}\left(z_{j}, r_{j} / 2\right)$, since in this case (5.7) gives

$$
|x-y| \geqq\left|y-z_{j}\right|-\left|x-z_{j}\right| \geqq r_{j}-r_{j} / 2=\delta\left|x_{1}-x_{2}\right| / 2^{j+4}
$$

for $j=i, i+1$. Compose the paths $\gamma_{i}$ into a single path $\varepsilon$ joining $x_{0}$ to $x_{1}$. It remains to show that $\varepsilon$ satisfies (5.5).

To this end let $x \in|\varepsilon|$. Then $x \in\left|\gamma_{i}\right|$ for some $i$. For $z \in\left|\gamma_{j}\right|$

$$
\begin{aligned}
\left|z-x_{1}\right| & \leqq\left|x_{1}-z_{j}\right|+\left|z-z_{j}\right| \leqq\left|x_{1}-z_{j}\right|+s / \delta 2^{j-1} \\
& \leqq s / 2^{j}+s / \delta 2^{j-1} \leqq 2 s / \delta 2^{j-1},
\end{aligned}
$$

since for $y=\infty$ (5.2) implies

$$
\left|z_{j}-z\right| \leqq\left|z_{j}-z_{j+1}\right| / \delta \leqq 2 s / \delta 2^{j} .
$$

Let now $z \in\left|\gamma_{j}\right|, j=i, i+1, \ldots$ be arbitrary. By (5.9) and (5.10)

$$
|z-x| \leqq\left|x-x_{1}\right|+\left|z-x_{1}\right| \leqq 4 s / \delta 2^{i-1} \leqq \operatorname{dist}(x, \complement D) / x,
$$

where $x=\delta^{4} 2^{-8}$. This proves (5.5). 
To prove (b) suppose that the domain $D \subset R^{n}$ is $(\alpha, \beta)$-uniform. Let $x_{1}, x_{2} \in D$, $x_{1} \neq x_{2}$. By Theorem 3.4, $D$ is of type $(\alpha, 2 \beta)$; hence there is a path $\gamma:[0, d] \rightarrow D$ joining $x_{1}$ to $x_{2}$ and satisfying (3.2) and (3.3). We shall show that $K=|\gamma|$ satisfies the condition (5.2), where $\delta$ depends only on $\alpha$ and $\beta$.

Let $x=\gamma(t), t \in(0, d), x \neq x_{1}, x_{2}$ and $y \in[D$. Suppose $t \leqq d / 2$. Assume first $\left|x_{2}-y\right| \leqq 4 \beta\left|x_{1}-x_{2}\right|$. Then

$$
\frac{|x-y|\left|x_{1}-x_{2}\right|}{\left|x-x_{1}\right|\left|x_{2}-y\right|} \geqq \frac{\alpha p_{d}(t)\left|x_{1}-x_{2}\right|}{\beta t\left|x_{2}-y\right|} \geqq \frac{\alpha}{4 \beta^{2}} .
$$

If $\left|x_{2}-y\right|>4 \beta\left|x_{1}-x_{2}\right|$, then

$$
\frac{|x-y|}{\left|x_{2}-y\right|} \geqq \frac{\left|y-x_{2}\right|-\left|x-x_{2}\right|}{\left|x_{2}-y\right|} \geqq \frac{\left|y-x_{2}\right|-\beta\left|x_{1}-x_{2}\right|}{\left|x_{2}-y\right|} \geqq 3 / 4,
$$

since the map $s \mapsto\left(s-\beta\left|x_{1}-x_{2}\right|\right) / s$ has the minimum $3 / 4$ in $\left[4 \beta\left|x_{1}-x_{2}\right|, \infty\right)$. On the other hand,

and thus

$$
\frac{\left|x_{1}-x_{2}\right|}{\left|x-x_{1}\right|}>\frac{\left|x_{1}-x_{2}\right|}{\beta\left|x_{1}-x_{2}\right|}=1 / \beta
$$

$$
\frac{|x-y|}{\left|x-x_{1}\right|} \frac{\left|x_{1}-x_{2}\right|}{\left|x_{2}-y\right|} \geqq 3 / 4 \beta \text {. }
$$

Thus we have a lower bound for the cross ratio $\left(x, y, x_{1}, x_{2}\right)$ in terms of $\alpha$ and $\beta$ whenever $y \in[D$ and $x=\gamma(t), t \leqq d / 2$. Observe that the case $y=\infty$ is trivial.

Next consider $\left(x, y, x_{2}, x_{1}\right)$ for $y \in \mathcal{C} D$ and $x=\gamma(t), t \leqq d / 2$. Assume first $|x-y| \geqq$ $c\left|y-x_{1}\right|, c=\alpha / \beta(1+\alpha / \beta)$. Now

$$
\left(x, y, x_{2}, x_{1}\right) \geqq c\left|x_{1}-x_{2}\right| /\left|x-x_{2}\right| \geqq c\left|x_{1}-x_{2}\right| / \beta\left|x_{1}-x_{2}\right|=c / \beta .
$$

If $|x-y|<c\left|y-x_{1}\right|$, then

$$
\left|x_{1}-x\right| \geqq\left|y-x_{1}\right|-|y-x| \geqq\left|y-x_{1}\right|-c\left|y-x_{1}\right|=\left|y-x_{1}\right| /(1+\alpha / \beta)
$$

and hence

$$
|y-x| \geqq \alpha t / \beta \geqq \alpha\left|x-x_{1}\right| / \beta \geqq c\left|y-x_{1}\right|,
$$

a contradiction. Thus (5.11) holds in each case.

To complete the proof we observe that the above estimates also hold, by symmetry, in the case $x=\gamma(t), d / 2 \leqq t \leqq d$. Hence we have the required lower bounds for the cross ratios in (5.2).

5.12. Remark. It is also possible to give alternative characterizations for uniform domains in terms of cross ratios. For instance, a domain $D \subset \bar{R}^{n}$ can be called $t$-uniform, $0<t \leqq 1$, if for all $x_{1}, x_{2} \in D, x_{1} \neq x_{2}$, there is a path $\gamma:[0,1] \rightarrow D$ joining $x_{1}$ to $x_{2}$ such that for all $z_{1}, z_{2} \in|\gamma|, z_{1} \neq z_{2}$ and $x \in|\gamma|$ between $z_{1}$ and $z_{2}$ the estimate $\left(x, y, z_{1}, z_{2}\right) \geqq t$ holds for all $y \in \mathcal{C} D$. To prove that this gives essentially the same concept as 5.1 requires lengthy technical constructions, which we omit here. Observe that this definition is also a conformal invariant in $\bar{R}^{n}$. 


\section{Properties of uniform domains}

6.1. Quasiconformal invariance. It was shown in [MS, Theorem 2.15] that if a domain $D \subset R^{n}$ is $(\alpha, \beta)$-uniform and $f: R^{n} \rightarrow R^{n}$ is a $K$-quasiconformal mapping, then $f D$ is $\left(\alpha^{\prime}, \beta^{\prime}\right)$-uniform and $0<\alpha^{\prime} \leqq \beta^{\prime}<\infty$ depend only on $\alpha, \beta, K$ and $n$. Since the cross ratio is invariant under Möbius transformations of $\bar{R}^{n}$, standard modulus estimates and a compactness argument give the following result.

6.2. Theorem. There is a function $\varphi_{n}:(0,1] \times[1, \infty) \rightarrow(0,1]$ depending only on $n$ such that

$$
\varphi_{n}(\delta, 1)=\delta=\lim _{K \searrow 1} \varphi_{n}(\delta, K),
$$

and if $D \subset \bar{R}^{n}$ is a $\delta$-uniform domain and $f: \bar{R}^{n} \rightarrow \bar{R}^{n}$ is $K$-quasiconformal, then $f D$ is $\varphi_{n}(\delta, K)$-uniform.

6.3. Remark. For $n=2$, because of Teichmüller's famous theorem, the change of the cross ratio under a quasiconformal map is known. Consequently, for $n=2$ the function $\varphi_{n}(\delta, K)$ can be calculated.

6.4. Hausdorff dimension of $\partial D$. It was proved in [GV] that if $f: \bar{R}^{n} \rightarrow \bar{R}^{n}$ is a quasiconformal mapping, then the Hausdorff dimension, $\operatorname{dim}_{H}$, of $f S^{n-1}$ satisfies $\operatorname{dim}_{H} f S^{n-1} \leqq c<n$, where $c$ depends only on $n$ and $K$. Observe that $\operatorname{dim}_{H} f S^{n-1}$ can take values arbitrarily close to $n$. Thus the following theorem is sharp.

6.5. Theorem. Suppose that $D \subset \bar{R}^{n}$ is a $\delta$-uniform domain. Then $\operatorname{dim}_{H} \partial D \leqq$ $c<n$, where $c$ depends only on $\delta$ and $n$.

Proof. For the proof we apply [S, Theorem 3.2]. Let $A \subset R^{n}$ and $z \in A$. Write

$$
G(z, A)=\varliminf_{r \rightarrow 0} \sup _{x \in B^{n}(z, r)} \operatorname{dist}(x, A) / r
$$

and $G(A)=\inf \{G(z, A): z \in A\}$. If $G(A)>0$, then $\operatorname{dim}_{H} A \leqq c<n$, where $c$ depends only on $G(A)$ and $n$; see [S, Theorem 3.2]. Thus it suffices to find a lower bound for $G(\partial D)$ depending only on $\delta$.

We may assume $\partial D \cap R^{n} \neq \emptyset$. Fix $z \in \partial D \cap R^{n}$ and choose $r_{0}>0$ such that for all $r \in\left(0, r_{0}\right], S^{n-1}(z, r) \cap D \neq \emptyset$.

Let $x_{1} \in S^{n-1}(z, r) \cap D, r \in\left(0, r_{0}\right]$. Pick $x_{2} \in D \cap S^{n-1}(z, r / 4)$. Since $D$ is $\delta$-uniform, there is a continuum $K$ joining $x_{1}$ to $x_{2}$ and $\left(x, y, x_{1}, x_{2}\right) \geqq \delta$ for all $x \in K \backslash\left\{x_{1}, x_{2}\right\}$ and $y \in \complement D$. Fix $x \in S^{n-1}(z, 3 r / 4) \cap K$ and then $y \in \complement D$ such that $|y-x|=\operatorname{dist}(\complement D, x)$. Now either $|y-x| \geqq r / 16$ or $|y-x|<r / 16$, in which case

Hence in both cases

$$
|y-x| \geqq \delta \frac{\left|x-x_{1}\right|\left|y-x_{2}\right|}{\left|x_{1}-x_{2}\right|} \geqq \delta \frac{(r / 4)(3 r / 4-r / 16-r / 4)}{r+r / 4}>\frac{r \delta}{16}
$$

$$
\sup _{x \in B^{n}(z, r)} \frac{1}{r} \operatorname{dist}(x, \partial D) \geqq \delta / 16, \quad r \in\left(0, r_{0}\right] .
$$

This is the required lower bound. 
6.6. A metric property. If $D \subset \bar{R}^{n}$ is a $\delta$-uniform domain, it is easy to show that $D$ is locally connected at boundary points (for the case $n=2$, see [MS, Lemma 2.29]). F. Gehring, see e.g. [G], has introduced an important metric property called $b$-locally connectedness. We recall the definition. A set $E \subset \bar{R}^{n}$ is said to be $b$-locally connected $1 \leqq b<\infty$ if, for all $z \in R^{n}$ and $r>0$, points in $E \cap \bar{B}^{n}(z, r)$ can be joined in $E \cap \bar{B}^{n}(z, b r)$ and points in $E \backslash B^{n}(z, r)$ can be joined in $E \backslash B^{n}(z, r / b)$.

6.7. Theorem. Suppose that $D \subset \bar{R}$ is a $\delta$-uniform domain. Then $D$ is b-locally connected and $b$ depends only on $\delta$.

Proof. Let $z \in D \cap R^{n}$ and $r>0$. Since $\delta$-uniform domains are invariant under the inversion in a ball, it suffices to show that points in $D \cap \bar{B}^{n}(z, r)$ can be joined in $D \cap \bar{B}^{n}(z, b r)$, where $b$ depends only on $\delta$. Let $x_{1}, x_{2} \in D \cap \bar{B}^{n}(z, r)$. Since $D$ is $\delta$-uniform, there is a continuum $K$ joining $x_{1}$ to $x_{2}$ in $D$ and satisfying (5.2). Set $b=1+5 / \delta$ and $S=S^{n-1}(z, b r)$. If $K \subset \bar{B}^{n}(z, b r)$ or $S \subset D$, we have proved the claim. Otherwise let $x \in K \cap S$ and $y \in \complement D \cap S$. Now (5.2) yields

$$
\frac{5}{\delta} r \leqq\left|x-x_{1}\right| \leqq \frac{1}{\delta} \frac{|x-y|\left|x_{1}-x_{2}\right|}{\left|y-x_{2}\right|} \leqq \frac{1}{\delta} \frac{2 b r 2 r}{(b-1) r}=\frac{4}{5}\left(1+\frac{5}{\delta}\right) r,
$$

clearly a contradiction since $\delta \in(0,1]$. This proves the theorem.

\section{References}

[G] Gehring, F.W.: Univalent functions and the Schwarzian derivative. - Comment. Math. Helv. 52, 1977, 561- 572.

[GV] Gehring, F. W., and J. VÄISÄLÄ: Hausdorff dimension and quasiconformal mappings.

- J. London Math. Soc. (2) 6, 1973, 504-512.

[MS] Martio, O., and J. SARvas: Injectivity theorems in plane and space. - Ann. Acad. Sci. Fenn. Ser. A I 4, 1978/1979 384-401.

[S] Sarvas, J.: The Hausdorff dimension of the branch set of a quasiregular mapping. - Ann. Acad. Sci. Fenn. Ser. A I 1, 1975, 297-307.

University of Helsinki

Department of Mathematics

SF-00100 Helsinki 10

Finland

Received 1 November 1979 